\title{
EXEMPLIFICATION OF RUIN PROBABILITIES
}

\author{
NiLS WiKsTAD \\ Stockholm
}

The following numerical values of ruin probabilities, $\Psi(u, T)$ for finite times $T$, have been calculated by the method proposed in "Analytical steps towards a numerical calculation of the ruin probability for a finite period when the risk process is of the Poisson type or of the more general type studied by Sparre Andersen", presented to this colloquium by Olof Thorin. The notations used in the sequel follow those of Thorin.

Two distributions of the individual claims are considered, viz.,

$$
\begin{array}{rlr}
\text { d.f. I: } P(y)=\mathrm{I} & -e^{-y} & , y \geq 0 \\
\text { d.f. II : } P(y)=\mathrm{I} & -0.0039793 e^{-0.014631 y}- & \\
& -0.1078392 e^{-0.190206 y}- & , y \geq 0
\end{array}
$$

The latter distribution is a rather crude attempt to interprete the extremely skew distribution (Swedish non-industry fire insurance I948-I95I) considered by Cramér in his treatise "Collective Risk Theory", Jubilee volume of Försäkringsaktiebolaget Skandia (I955) pp. 43-45.

Likewise two distributions of the interoccurence times are considered, viz.,
d.f. A : $K(t)=\mathrm{I}-e^{-t}$
d.f. B: $K(t)=\mathrm{I}-0.25 e^{-0.4 t}-0.75 e^{-2 t}$$$
, t \geq 0
$$$$
, t \geq 0
$$

The d.f.B was considered by Sparre Andersen (TICA I957 vol. II pp. 225-227).

Note that the first moment equals one in all the d.f. mentioned.

Though the analytical machinery also seems to work for $0 \leq c \leq \mathrm{I}$ the $\Psi$ values are indicated only for some values of $c>\mathrm{I}$. As known from Thorin's paper $c$ stands for $\mathrm{I}+\lambda$, where $\lambda$ is the premium 
loading, which means that for $c=0 \Psi(u, T)$ corresponds to the tail of the d.f. for the total amount of claims during the period $(0, T)$.

For comparison the value of the "insolvency constant" $R$ is also given.

In the computer program used there is a subroutine for finding the roots of a polynomial equation with complex coefficients, which routine originates from the IBM SSP (PL I) library but has been modified and rewritten in FORTRAN. The main algorithm is based on the Newton-Raphson method generalized by K. Nickel in ,Die numerische Berechnung der Wurzeln eines Polynoms", Numerische Mathematik vol. 9 (1966) pp. 80-98.

For the inversion we have at this stage used a method given by Piessens in "New Quadrature Formulas for Numerical Inversion of the Laplace Transform", BIT vol. 9 (I969) pp. 35I-36I. So far as possible we have tried to check the obtained values. However, we must admit the possibility of slighter differences from the exact values. Later a more careful study of such differences will be made.

The tables are labelled after the d.f. involved, i.e. table IA means that the claims have the d.f. I and the interoccurence times have the d.f. A. 
TABLE I A

\begin{tabular}{lccccc} 
& $U=$ & \multicolumn{3}{c}{$T=\mathrm{I}$} \\
$\mathrm{C}=1.05$ & .4698 & .2420 & .0003 & .0000 & .0000 \\
$\mathrm{C}=\mathrm{I} .10$ & .4634 & .2381 & .0003 & .0000 & .0000 \\
$\mathrm{C}=1.15$ & .4572 & .2342 & .0003 & .0000 & .0000 \\
$\mathrm{C}=1.20$ & .4510 & .2305 & .0003 & .0000 & .0000 \\
$\mathrm{C}=1.25$ & .4450 & .2268 & .0003 & .0000 & .0000 \\
$\mathrm{C}=1.30$ & $.439 \mathrm{I}$ & .2232 & .0003 & .0000 & .0000 \\
$\mathrm{C}=2.00$ & .3662 & .1800 & .0002 & .0000 & .0000
\end{tabular}

\begin{tabular}{lccccc} 
& $U=$ & \multicolumn{3}{c}{$T=$ I0 } \\
$\mathrm{C}=1.05$ & .8042 & .6376 & .0367 & .0000 & .0000 \\
$\mathrm{C}=1.10$ & .7854 & .6126 & .0319 & .0000 & .0000 \\
$\mathrm{C}=1.15$ & .7666 & .5882 & .0277 & .0000 & .0000 \\
$\mathrm{C}=1.20$ & .7477 & .5644 & .0241 & .0000 & .0000 \\
$\mathrm{C}=1.25$ & .7290 & .5414 & .0209 & .0000 & .0000 \\
$\mathrm{C}=1.30$ & .7105 & .5193 & .0182 & .0000 & .0000 \\
$\mathrm{C}=2.00$ & .4967 & .2993 & .0028 & .0000 & .0000
\end{tabular}

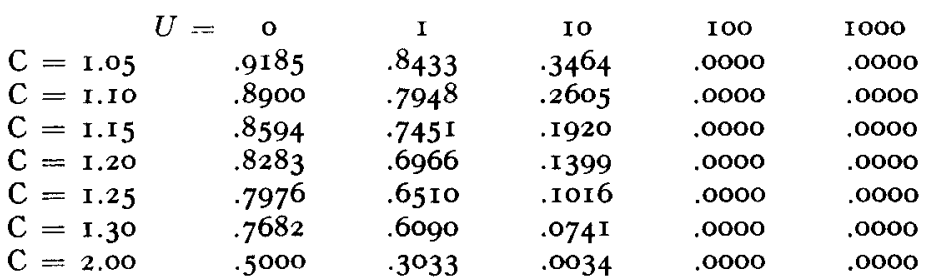

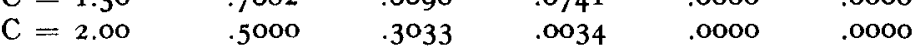

\begin{tabular}{lcccccc} 
& $U=$ & \multicolumn{7}{c}{$T=\infty$} \\
$\mathrm{C}=\mathrm{I} .05$ & .9524 & $.908 \mathrm{I}$ & .5916 & $.008 \mathrm{I}$ & .0000 & .0476 \\
$\mathrm{C}=\mathrm{I} .10$ & $.909 \mathrm{I}$ & $.830 \mathrm{I}$ & .3663 & $.000 \mathrm{I}$ & .0000 & .0909 \\
$\mathrm{C}=\mathrm{I} .15$ & .8696 & .7632 & .2360 & .0000 & .0000 & .1304 \\
$\mathrm{C}=\mathrm{I} .20$ & .8333 & .7054 & .1574 & .0000 & .0000 & .1667 \\
$\mathrm{C}=1.25$ & .8000 & .6550 & .1083 & .0000 & .0000 & .2000 \\
$\mathrm{C}=1.30$ & .7692 & .6107 & .0765 & .0000 & .0000 & .2308 \\
$\mathrm{C}=2.00$ & .5000 & .3033 & .0034 & .0000 & .0000 & .5000
\end{tabular}


TABLE I B

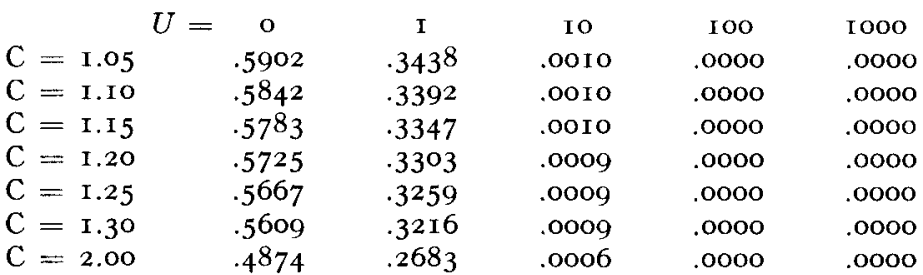

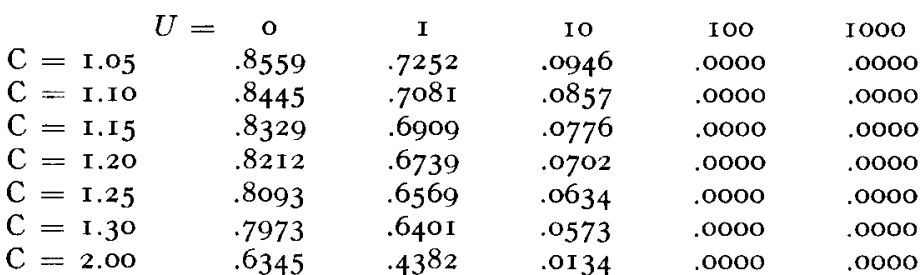

\begin{tabular}{|c|c|c|c|c|c|}
\hline & o & I & Io & 100 & IOOO \\
\hline $\mathrm{C}=\mathrm{I} .05$ & .9432 & .8890 & .4855 & .0000 & .0000 \\
\hline $\mathrm{C}=\mathrm{I} .10$ & $.927 \mathrm{I}$ & .8603 & .4117 & .0000 & .0000 \\
\hline$C=\mathrm{I.I5}$ & .9098 & .8299 & .3446 & .0000 & .0000 \\
\hline $\mathrm{C}=\mathrm{I} .20$ & .8915 & .7988 & .2857 & .0000 & .0000 \\
\hline$C=1.25$ & .8727 & .7678 & .2352 & .0000 & .0000 \\
\hline$C=1.3^{\circ}$ & .8539 & .7375 & $.193^{\circ}$ & .0000 & .0000 \\
\hline$C=2.00$ & .6417 & .4485 & .0178 & .0000 & .0000 \\
\hline
\end{tabular}

\begin{tabular}{|c|c|c|c|c|c|}
\hline & $\mathrm{o}$ & I & IO & IOO & 1000 \\
\hline $\mathrm{C}=1.05$ & .9686 & .9386 & $.703 \mathrm{I}$ & .0196 & .0000 \\
\hline $\mathrm{C}=\mathrm{I} . \mathbf{1 0}$ & $.945^{8}$ & .8959 & .5490 & .0029 & .0000 \\
\hline $\mathrm{C}=\mathrm{I} . \mathrm{I} 5$ & .9220 & .8528 & .4227 & .0003 & .0000 \\
\hline $\mathrm{C}=\mathrm{r} .2 \mathrm{O}$ & .8990 & .8127 & .3277 & .0000 & .0000 \\
\hline$C=1.25$ & $.877^{2}$ & .7759 & $.257 \mathrm{I}$ & .0000 & .0000 \\
\hline$=\mathbf{I} \cdot 3^{\circ}$ & .8565 & .7420 & .2040 & .0000 & .0000 \\
\hline$=2,00$ & .6417 & .4485 & .0178 & .0000 & .0000 \\
\hline
\end{tabular}

\begin{tabular}{|c|c|c|c|c|c|c|}
\hline & $U=$ & I & IO & 100 & IOOO & $R$ \\
\hline $\mathrm{C}=1.05$ & .9724 & .9459 & .7377 & .0614 & .0000 & .0276 \\
\hline$C=1.10$ & .9465 & $.897^{2}$ & .5542 & .0045 & .0000 & .0535 \\
\hline $\mathrm{C}=\mathrm{I} . \mathrm{I} 5$ & $.922 \mathrm{I}$ & $.853^{\circ}$ & $.4^{2} 31$ & .0004 & .0000 & .0779 \\
\hline $\mathrm{C}=\mathrm{I} .20$ & .8991 & $.8 \mathrm{I} 28$ & .3277 & .0000 & .0000 & .1009 \\
\hline$C=1.25$ & $.877^{2}$ & .7759 & $.257^{\circ}$ & .0000 & .0000 & .1228 \\
\hline $\mathrm{C}=\mathbf{1} \cdot 3^{\circ}$ & .8565 & .7420 & .2040 & .0000 & .0000 & .1435 \\
\hline$C=2.00$ & .6417 & .4485 & .0178 & .0000 & .0000 & $.35^{8} 3$ \\
\hline
\end{tabular}


TABLE II A

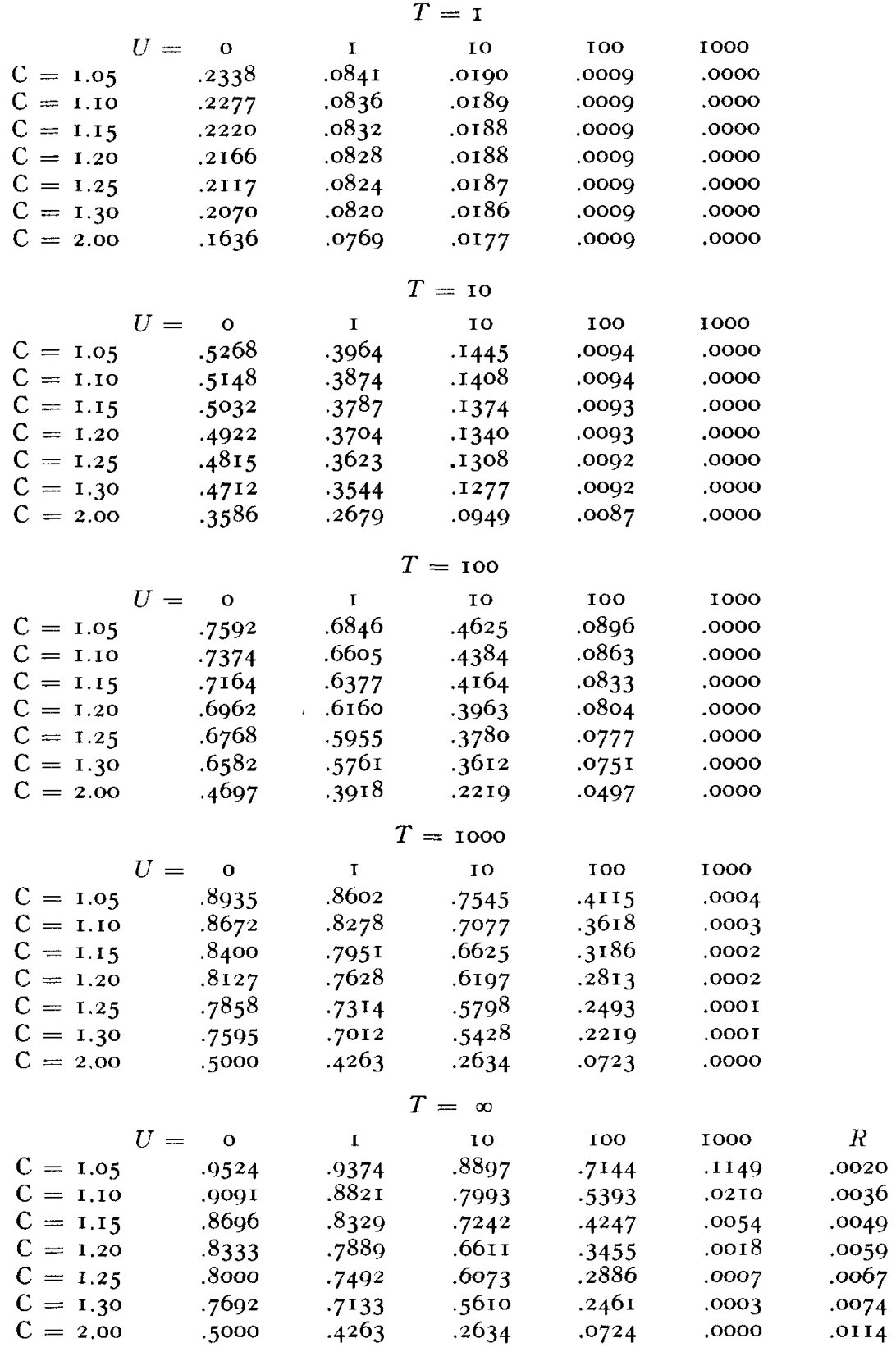


TABLE II B

\begin{tabular}{|c|c|c|c|c|c|}
\hline & \multicolumn{5}{|c|}{$T=\mathrm{I}$} \\
\hline & $U=$ & I & IO & 100 & 1000 \\
\hline$C=1.05$ & .3300 & . I 186 & .0276 & .0013 & .0000 \\
\hline$C=1.10$ & $.322 \mathrm{I}$ & .1180 & .0275 & .0013 & .0000 \\
\hline $\mathrm{C}=\mathrm{I} .15$ & .3146 & .1173 & .0274 & .0013 & .0000 \\
\hline$C=1.20$ & .3076 & .1167 & .0273 & .0013 & .0000 \\
\hline$C=1.25$ & .3010 &. $\operatorname{II6I}$ & $.027^{2}$ & .0013 & .0000 \\
\hline$C=1.3^{\circ}$ & $.294^{8}$ & .1155 & $.027 \mathrm{I}$ & .0013 & .0000 \\
\hline$C=2.00$ & .2352 & .1084 & .0257 & .0013 & .0000 \\
\hline
\end{tabular}

\begin{tabular}{|c|c|c|c|c|c|}
\hline & \multicolumn{5}{|c|}{$T=\mathbf{1 0}$} \\
\hline & $U=$ & I & 10 & 100 & 1000 \\
\hline $\mathrm{C}=\mathrm{I} .05$ & .5962 & .4345 & .1686 & .0103 & .0000 \\
\hline $\mathrm{C}=\mathrm{T} . \mathrm{IO}$ & .5845 & .4260 &. $\mathrm{I} 647$ & .0103 & .0000 \\
\hline $\mathrm{C}=\mathrm{I} . \mathrm{I} 5$ & $.573^{\mathrm{I}}$ & $.4^{17} 78$ & $.16 \mathrm{IO}$ & .0103 & .0000 \\
\hline $\mathrm{C}=\mathrm{I} .2 \mathrm{O}$ & .5622 & .4098 & .1574 & .0102 & .0000 \\
\hline$C=1.25$ & $.55^{\mathrm{I} 7}$ & .4021 &. $\mathbf{5} 539$ & .0102 & .0000 \\
\hline$C=1.30$ & .5415 & $.394^{6}$ & .1506 & .0101 & .0000 \\
\hline$C=2.00$ & .4280 & .3101 & .1145 & .0096 & .0000 \\
\hline
\end{tabular}

\begin{tabular}{lccccl} 
& $U=$ & \multicolumn{5}{c}{$T=$ I00 } \\
$\mathrm{C}=1.05$ & $.797^{8}$ & .7098 & .4898 & .0932 & I0 \\
$\mathrm{C}=\mathrm{I.10}$ & .7789 & .6878 & .4662 & .0898 & .0000 \\
$\mathrm{C}=\mathrm{I.15}$ & .7605 & .6667 & .4444 & .0867 & .0000 \\
$\mathrm{C}=\mathrm{1.20}$ & .7426 & .6466 & .4244 & .0837 & .0000 \\
$\mathrm{C}=1.25$ & .7253 & .6275 & .4059 & .0809 & .0000 \\
$\mathrm{C}=1.30$ & .7086 & .6094 & .3889 & .0782 & .0000 \\
$\mathrm{C}=2.00$ & .5308 & .4314 & .2439 & .0519 & .0000
\end{tabular}

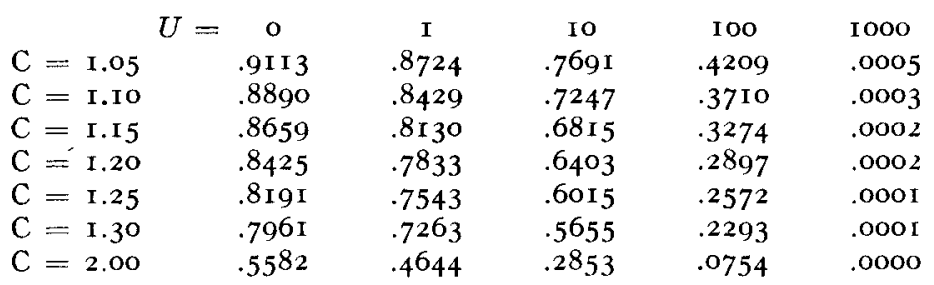

\begin{tabular}{|c|c|c|c|c|c|c|}
\hline & $U=$ & I & 10 & 100 & 1000 & $R$ \\
\hline$C=1.05$ & .9608 & .9436 & .8975 & .7231 & .1225 & .0020 \\
\hline $\mathrm{C}=\mathrm{I} . \mathrm{IO}$ & .9247 & .8933 & .8125 & $.55^{\circ 2}$ & .0232 & .0035 \\
\hline$C=1.15$ & $.89 \times 3$ & .8484 & .7412 & .4356 & $.006 \mathrm{r}$ & .0047 \\
\hline$C=1.20$ & .8603 & .8079 & .6806 & .3557 & .0020 & .0057 \\
\hline$C=1.25$ & .8315 & .7712 & .6286 & $.297^{8}$ & .0008 & .0066 \\
\hline $\mathrm{C}=\mathrm{I} .3^{\circ}$ & .8047 & .7378 & $.5^{835}$ & .2544 & .0004 & .0073 \\
\hline$C=2.00$ & $.55^{82}$ & .4645 & .2853 & .0754 & .0000 & .0113 \\
\hline
\end{tabular}

